\title{
ATIVIDADE DA ÁCIDO DELTA-AMINOLEVULÍNICO-DESIDRATASE NA POPULAÇÃO URBANA DO MUNICIIPIO DE LONDRINA, PARANÁ 1
}

\author{
LEDA MEZZAROBA ${ }^{2}$ \\ CONCEIÇÃOA. TURINI² \\ MONICAM. B. PAOLIELO? \\ MARIA INÉS REZENDE \\ TIEMI MATSUO 3
}

MEZZAROBA, L.; TURINI, C. A.; PAOLIELO, M. M. B.; REZENDE, M. I.; MATSUO, T. Atividade da ácido delta-aminolevulínico-desidratase na população urbana do município de Londrina, Paraná. Semina: Ci. Biol. Saúde, Londrina, v. 20/21, n. 2, p. 73-79, jun. 1999/2000.

\begin{abstract}
RESUMO: A contaminação do solo, água, ar e alimentos por metais pesados, em decorrência de processos industriais, tem atingido não somente trabalhadores, mas também a população em geral. A determinação da atividade da ácido $\delta$-aminolevulínico desidratase ( $A L A-D$ ) é o mais sensivel indicador biológico de efeito subcritico ao chumbo. O objetivo deste estudo foi avaliar a atividade da ALA-D em uma amostra populacional urbana do Municipio de Londrina, Paraná. A população estudada foi constituida por 87 sujeitos (52 mulheres e 37 homens) selecionados a partir de uma amostra inicial de 120 voluntários, de acordo com critérios de exclusão previamente estabelecidos. Foram excluidos os indivíduos expostos ocupacionalmente ao chumbo, com exames clínico-laboratoriais alterados, obesos, hipertensos, diabéticos e fumantes, cujas residências se localizavam próximas às áreas de utilização do metal. Os valores de referência (VR) obtidos para a atividade da ALA-D foram de 21,33-59,90 U/L, não se encontrando diferenças significativas entre os valores para a população masculina e feminina. Estes resultados se mostraram semelhantes aos descritos em outros trabalhos, que também utilizaram amostras populacionais e mesma metodologia analitica. A padronização do uso de bioindicadores para a monitorização das populações expostas a poluentes ambientais, representa uma das medidas mais úteis para o diagnóstico precoce e prevenção das intoxicações por chumbo em áreas urbanas.
\end{abstract}

PALAVRAS-CHAVE: ácido $\delta$-aminolevulínico desidratase; monitorização ambiental; chumbo; toxicologia.

\section{INTRODUÇÃO}

Nos últimos anos, as populações urbanas expostas a poluentes ambientais têm sido objeto de numerosas pesquisas, especialmente nos paises industrializados. Entre estes estudos, destacaram-se as investigações a respeito dos efeitos tóxicos produzidos à saúde humana por metais pesados, como o chumbo, resultantes não apenas da exposição de trabalhadores em processos extrativos e industriais, mas também da população urbana em geral, através do solo, da água, do ar e da ingestão de alimentos contaminados (Larini, 1993; Salgado, 1996).

A determinação da plumbemia é um indicador biológico de exposição bastante utilizado para grupos de indivíduos expostos ocupacionalmente porque as concentrações sangüineas de chumbo apresentam uma correlação positiva com os niveis do metal no ambiente de trabalho (Salgado, 1994).

A legislação brasileira, através Norma Regulamentadora NR-7 - Portaria $n^{\circ} 24$ - da Secretaria de Segurança e Saúde no Trabalho, de 29 de dezembro de 1994, prevê como indicadores biológicos para exposições ao chumbo a dosagem de chumbo em sangue ( $\mathrm{Pb}-\mathrm{S}$ ), a determinação de ácido delta-aminolevulínico na urina (ALA-U) e da zinco-protoporfirina em sangue (ZPP-S). Essa norma estabelece como parâmetros para exposições ocupacionais ao chumbo o Valor de Referência de Normalidade (VR) e o Índice Biológico Máximo Permitido (IBMP) de $40 \mu \mathrm{g} / \mathrm{dl}$ e $60 \mu \mathrm{g} / \mathrm{dl}$, respectivamente (Della Rosa, Siqueira \& Colacioppo, 1996)

\footnotetext{
${ }^{1}$ Setor de Toxicologia, Laboratório de Análises Clínicas, Hospital Universitário Regional do Norte do Paraná.

${ }^{2}$ Docentes do Departamento de Patologia Aplicada, Legislação e Deontologia, Centro de Ciências da Saúde, Universidade Éstadual de Londrina, Paraná.

${ }^{3}$ Docente do Departamento de Matemática Aplicada, Centro de Ciências Exatas, Universidade Estadual de Londrina.
} 
Embora as intoxicações por chumbo tenham quadros clínico e bioquímico bem estabelecidos (Ellenhorn, 1997), um dos problemas ainda não solucionados se refere às alterações funcionais. inclusive moleculares, causadas por concentrações baixas de chumbo no sangue. Resultados de diferentes pesquisas revelaram que, em concentrações de $20 \mu \mathrm{g} / \mathrm{dl}$ de sangue total já ocorrem efeitos nocivos sobre o sistema hematopoiético humano, como a interferência na síntese do Heme, pela inibição da atividade da enzima ácido delta-aminolevulínico desidratase ou ALA-D (Salgado, Fonseca \& Lepera, 1985,; Garcia Fernandez, 1989; CDC, 1991; Wananukul, 1998).

A determinação da atividade da ALA-D é considerado o mais sensivel indicador biológico de efeito subcrítico ao chumbo, muito útil para o diagnóstico precoce e prevenção das intoxicações pelo metal, porque apresenta uma excelente correlação negativa com níveis de chumbo na faixa de 10 a $60 \mu \mathrm{g} / \mathrm{dl}$ (Salgado, 1996). Segundo Sakai \& Morita (1996), os níveis de ácido deltaaminolevulínico plasmático (ALA-P) nas células sangüineas aumentam mais lentamente em concentrações de chumbo menores que $40 \mu \mathrm{g} / \mathrm{dl}$ de sangue total ("slow phase") e rapidamente em concentrações superiores a $40 \mu \mathrm{g} / \mathrm{dl}$ de sangue total ("rapid phase").

Quanto aos estudos de populações não expostas ocupacionalmente ao chumbo, destacaram-se, no Brasil, os trabalhos pioneiros de Fernícola \& Azevedo (1981) sobre as correlações existentes entre os valores de plumbemia e a atividade da ALA-D e de Sousa, Porto \& Azevêdo (1987) que propuseram valores de referência de ALAD para a população urbana, sem história de exposições profissionais.

De acordo com os resultados destas e de outras pesquisas, em concentrações sangüíneas de $10 \mathrm{a}$ $20 \mu \mathrm{g}$ de chumbo/dl de sangue total, a inibição da ALA-D leva ao aumento do ALA-P, e, por conseqüência, eleva-se a excreção deste metabólito na urina. Além dos efeitos deletérios resultantes da exposição ao chumbo, especialmente sobre a medula óssea, o sistema nervoso e os rins, o aumento de ALA-P, um precursor do HEME acumulado também na porfiria intermitente, tem sido relacionado à sindrome encéfalo-polineurítica. $A$ encefalopatia que se desenvolve nas exposições a longo prazo ao chumbo compreende alterações subclínicas sensoriais, perceptuais e das funções psicomotoras detectáveis através de testes fisiológicos e psicofisiológicos (Salgado, 1996).

Outras conseqüências do acúmulo de ALA-P no organismo humano foram também detectadas por Costa et al.(1997). Segundo os autores, existe uma associação entre o acúmulo de ALA-P e a elevação da atividade da superóxido-desmutase (SOD) que ocorre em diversas patologias relacionadas ao "stress oxidativo", entre elas, as intoxicações por chumbo e mercúrio. Os resultados de seus trabalhos forneceram evidências de que a distribuição e o acúmulo de ALA-P no organismo humano pode ser correlacionado a diferentes respostas de "stress oxidativo", como o aumento da atividade enzimática da SOD eritrocitária, aumento da oxidação da hemoglobina a metahemoglobina superóxido, além da elevação da protoporfirina IX (PP-IX) em trabalhadores expostos ao chumbo

Deste modo, o estabelecimento do valor de referência para ALA-D (VR definido por Apostoli \& Minoia, em 1991, como " o valor obtido por meio da observação ou quantidade particular em um indivíduo ou na população de referência"), representa o início de uma adequada monitorização da exposição ambiental de uma população ao chumbo. No Brasil, a falta de bases de dados para obtenção de valores de referência leva à "reprodução" dos valores obtidos em outros países, muitas vezes inadequados à nossa realidade (Salgado, 1990; Cordeiro \& LimaFilho, 1995; Paolielo et al., 1997).

O objetivo geral deste estudo foi o de avaliar a atividade da ALA-D em uma amostra da população urbana do Município de Londrina, Paraná. Para isso, pretendeu-se comparar os dados obtidos com os valores de referência desta enzima disponiveis na literatura especializada. Com esses resultados, espera-se contribuir para a padronização da metodologia analitica e dos valores de referência de ALA-D necessários à detecção precoce dos efeitos nocivos à saúde, decorrentes da exposição ambiental das comunidades urbanas brasileiras aos derivados orgânicos e inorgânicos do chumbo.

\section{MATERIAL E MÉTODOS}

\section{Estabelecimento da população de referência e critérios de exclusão}

Para estabelecer a população de referência da pesquisa foram previamente definidos alguns parâmetros de condições de exposição ao chumbo e de condições de saúde da população em estudo. No que se refere às condições de exposição, a seleção obedeceu aos seguintes critérios de exclusão: indivíduos não expostos ocupacionalmente ou por "hobby" ao chumbo inorgânico, cujas 
residências eram distantes de locais de utilização do metal (lengar \& Wolttiez, 1988; Minoia et al., 1990). Estas informações foram obtidas através da aplicação de um questionário para levantamento de dados pessoais (idade, sexo, grupo racial, ocupação, local de moradia nos últimos três anos, intensidade de tráfego na área de residência, entre outros), dados relativos a hábitos pessoais (consumo de bebidas alcoólicas e tabagismo) e alimentares (tipo de dieta, consumo de água e bebidas não alcoólicas).

Para avaliar as condições de saúde da população em estudo, realizou-se o exame clínico de cada um dos indivíduos. Esta consulta incluiu anamnese, aferição da pressão arterial, do peso corpóreo e solicitação dos seguintes exames laboratoriais complementares: hemograma, glicose, uréia, creatinina, gama glutamil-transferase, alanina aminotransferase, aspartato amino-transferase, colesterol total, triglicérides e urina tipo 1.

Embora a exposição ao chumbo tenha sido considerado o principal critério para a seleção dos participantes, foram excluidos também os individuos que apresentaram valores clínicolaboratoriais fora dos valores referenciais preconizados pela literatura nas diferentes metodologias analíticas utilizadas na pesquisa. Estas pessoas receberam encaminhamento para consultas e/ou exames complementares, necessários ao tratamento das patologias detectadas e que motivaram sua exclusão do presente estudo.

A influência do hábito de fumar sobre a plumbemia e, conseqüentemente, sobre a atividade da ALA-D, citada em trabalhos anteriores sobre o tema (Fernícola \& AzevedO, 1981; Sousa et al., 1987), justificou a exclusão dos fumantes de $10 \mathrm{ou}$ mais cigarros de tabaco/dia. Foram excluídos também os obesos, os hipertensos e aqueles afetados por doenças crônicas como diabetes, distúrbios cardiovasculares e alcoolismo (Bortoli et al., 1986; lengar \& Wolttiez, 1988; Minoia et al., 1990; Wider et al., 1997).

A população estudada foi constituída por um grupo de 87 indivíduos, com idade que variou entre 15 e 65 anos, considerados "sadios" (52 mulheres e 35 homens), selecionados a partir de uma amostra inicial de 120 sujeitos, de acordo com os critérios de exclusão previamente estabelecidos.

Os contatos para o recrutamento de voluntários das várias regiões da área urbana (central e periférica) do Município de Londrina, Paraná, foram feitos com o auxílio de associações de bairros, instituições religiosas, conselhos regionais de saúde e instituições públicas de ensino e de saúde
(Unidades Básicas de Saúde e hospitais da rede pública de saúde).

Estas pessoas foram antecipadamente orientadas quanto às condições adequadas às colheitas de sangue e urina (jejum e abstinência no consumo de bebidas alcoólicas nas últimas 24 horas) e aos outros procedimentos clínicos que foram realizados em finais de semana, principalmente em escolas e igrejas, no periodo compreendido entre novembro de 1994 a dezembro de 1996

\section{Procedimento analítico}

As amostras de sangue foram coletadas em sistemas a vácuo apropriados a cada tipo de exame laboratorial solicitado e acondicionadas em banho de gelo para o transporte até o Laboratório de Análises Clínicas do Hospital Universitário Regional do Norte do Paraná, Universidade Estadual de Londrina (LAC/HURNP/UEL), onde foram imediatamente processadas.

A atividade da ALA-D foi determinada pelo Método Padronizado Europeu (Berlin \& Schaller, 1974) que se baseia na incubação da enzima com excesso de ácido delta-aminolevulínico: o porfobilinogênio produzido, na presença do Reagente de Ehrlich, desenvolve coloração rósea que é medida espectrofotometricamente. Os resultados são expressos em U/L de ALA/minuto/litro de eritrócito, com o hematócrito determinado em contador automatizado de células.

\section{Análise estatística}

Os resultados referentes à atividade da ALA-D na amostra selecionada receberam o seguinte tratamento estatístico:

a) Valor mínimo, $5^{\circ}$ percentil, $1^{\circ}$ quartil, mediana, $3^{\circ}$ quartil, $95^{\circ}$ percentil, valor máximo, média geométrica, média aritmética e desvio-padrão;

b) Intervalo de confiança (95\%) para o valor médio $\overrightarrow{\mathrm{x}} \pm \mathrm{t}_{[a: n-1]} . \mathrm{s}_{\overline{\mathrm{x}}}$ onde $\overline{\mathrm{x}}$ é o valor médio, $\mathrm{t}_{[a: n-1]}$ é o valor da distribuição t de Student e $s_{\bar{x}}$ é o erro padrão da média;

c) Intervalo experimental delimitado pelo menor e maior valores observados $\left(x_{\min }\right.$ e $x_{\max }$, respectivamente);

d) Valor de referência obtido através do intervalo $\overline{\mathrm{X}} \pm 2$.s , onde $\overline{\mathrm{X}}$ é o valor médio e $s$ é o desviopadrão dos valores observados. Se o menor valor observado $\left(x_{\text {min }}\right)$ for maior do que $\overline{\mathrm{x}}-2 . \mathrm{s}, \mathrm{o}$ intervalo será delimitado pelos valores $x_{m i n} \mathrm{e}$ $\overline{\mathrm{x}}+2 . \mathrm{s}$; 
e) Intervalo de incerteza que é o intervalo dos altos valores observados, delimitado por $\bar{X}+2 . s$ e o valor máximo obtido $\left(x_{\text {imin }}\right)$.

\section{RESULTADOS E DISCUSSÃO}

Dos 120 voluntários examinados, 20 foram excluidos em função de alterações clínicolaboratoriais e 13 por serem fumantes $(27,5 \%$ de exclusão). Assim, 87 sujeitos participaram da pesquisa, sendo 52 do sexo feminino e 35 do sexo masculino.

A Tabela abaixo demonstra as estatísticas para os valores de ALA-D em indivíduos considerados saudáveis, segundo o sexo.

Como se pode observar, os valores de referência
(VR) obtidos no presente estudo para a atividade da ALA-D foram de 21,33-59,90 U/L na população estudada. Não se observou diferença significativa entre os valores de ALA-D encontrados na população masculina e feminina participante do estudo ( $t=54,9$ e $p=0,00620)$.

Os dados disponiveis na literatura consultada indicaram que, individuos saudáveis e não expostos ocupacionalmente ao chumbo, apresentaram valores de referência para atividade da ALA-D maiores que 20 U/L (Berlin \& Schaller, 1974; Garcia Fernández et al., 1989). De acordo com os resultados apresentados por Fernícola \& Azevedo (1981) os valores de atividade da ALA-D para a população urbana da Grande São Paulo foram de 20,8-67,6 U/L. Em pesquisas semelhantes, Sousa et al. (1987) determinaram que a média global da atividade enzimática na população de Salvador

Tabela - Valores de atividade de ALA-D (U/L) em sujeitos saudáveis, segundo o sexo, Município de Londrina, Paraná

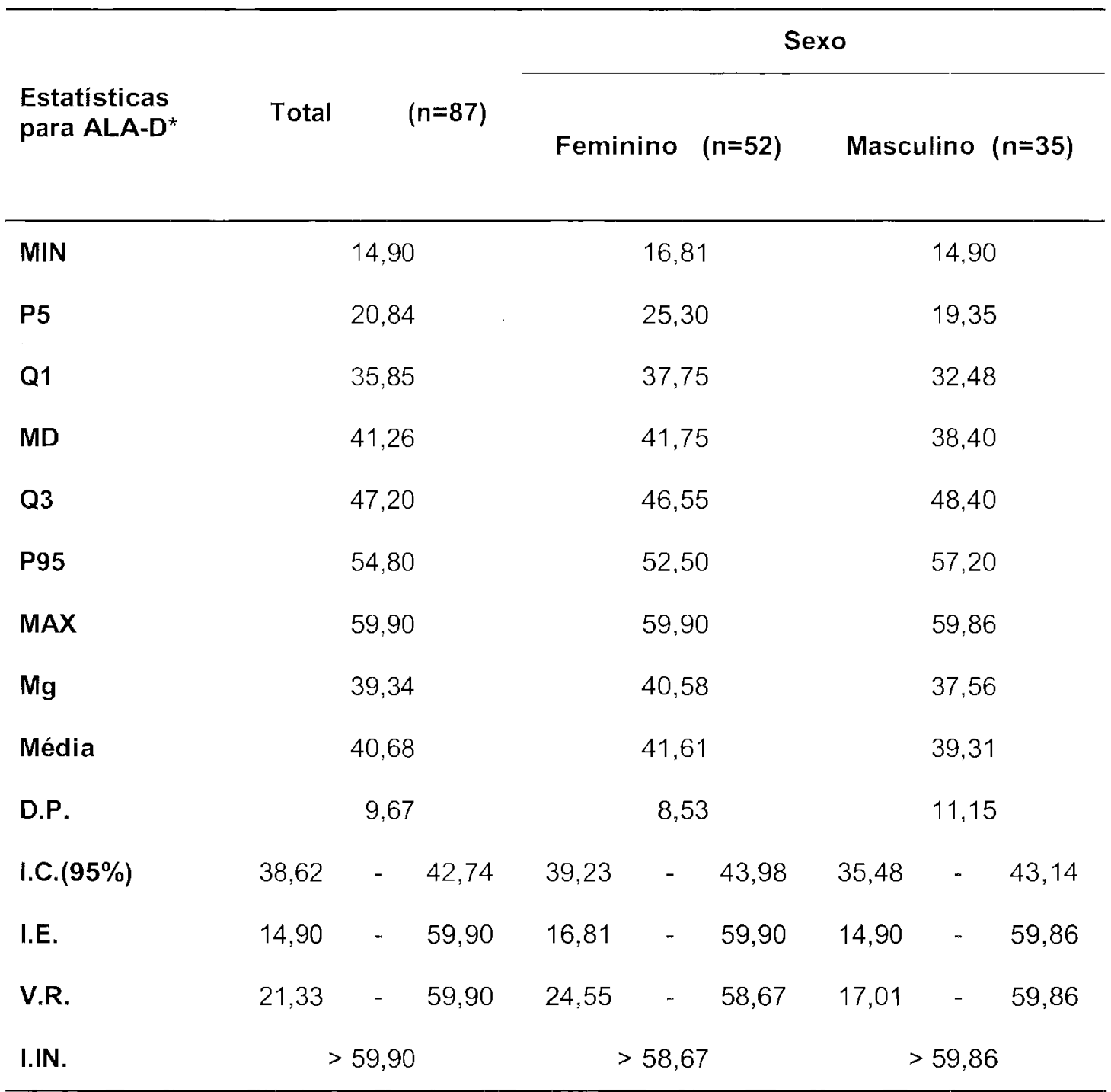

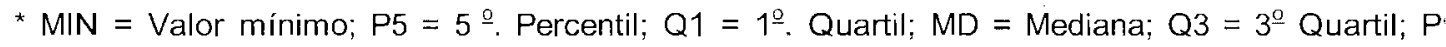
Percentil; MAX = Valor máximo; $\mathrm{Mg}=$ Média geométrica; Média = Média aritmética; $D$.P. = Desvic I.C. $(95 \%)=$ Intervalo de Confiança $(95 \%) ;$ I.E. = Intervalo experimental; $V . R .=$ Valores de referênci Intervalo de incerteza. 
(Bahia) foi de $34,5 \pm 14,5 \mathrm{U} / \mathrm{L}$, variando os valores de 9,0 até 80,0 U/L e, na região sul do Estado de Minas Gerais, Siqueira \& Pessoa (1999) obtiveram resultados de $54,52 \pm 9,80 \mathrm{U} / \mathrm{L}$.

Deste modo, os valores de referência obtidos no presente estudo $(21,33-59,90 \mathrm{U} / \mathrm{L})$ se mostraram semelhantes aos resultados de trabalhos que também utilizaram amostras populacionais urbanas e a mesma metodologia analítica.

De acordo com Sousa et al. (1987), a atividade da ALA-D pode variar consideravelmente de uma região para outra e entre indivíduos, em função de diferentes fatores como a poluição atmosférica e o chumbo absorvido com os alimentos. Ainda segundo os autores, a não-uniformização do método laboratorial usado para determinação da atividade enzimática também dificulta a comparação entre os resultados de pesquisas realizadas em diferentes regiões geográficas do Brasil.

Na Figura apresentada a seguir, pode-se visualizar a distribuição homogênea dos valores de ALA-D encontrados, segundo o sexo, através de gráficos "box-plot" ou "gráficos dos cinco números" que são os valores mínimo e máximo obtidos, o primeiro quartil ou $25 \%$, a mediana ou segundo quartil e o terceiro quartil ou $75 \%$.

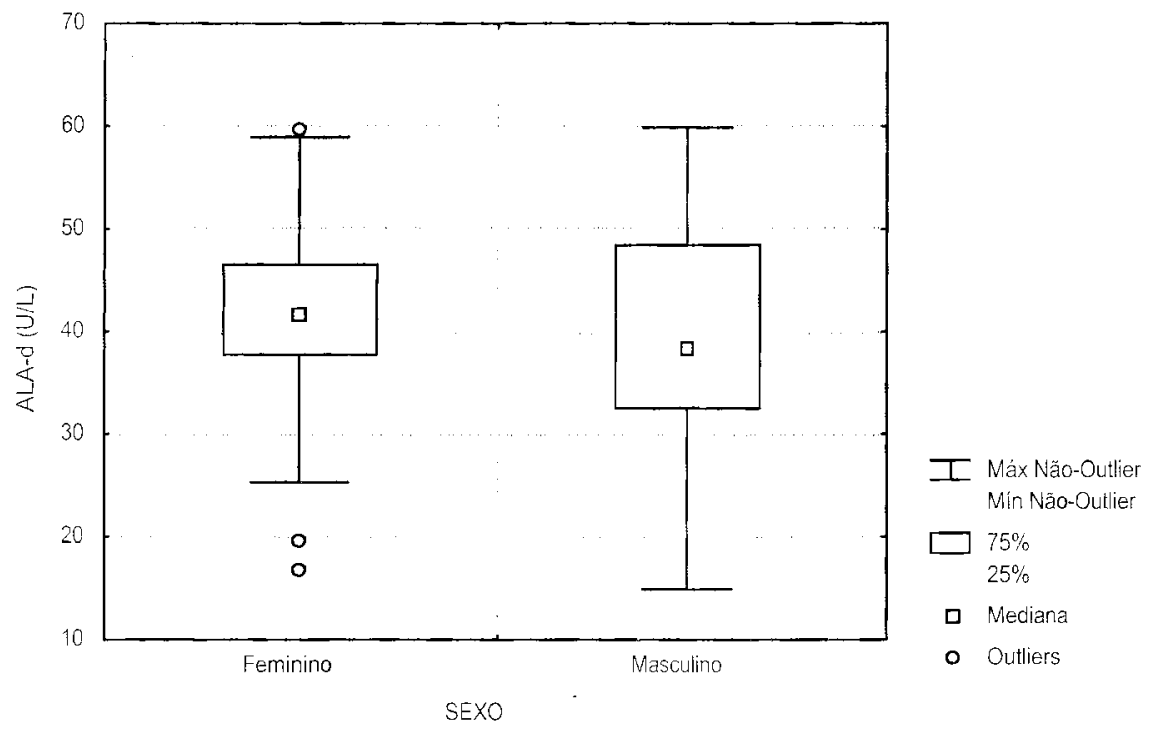

Figura - "Box-and-whisker plot" para atividade de ALA-D (U/L), segundo o sexo, Municipio de Londrina, Paraná.

Apesar da importância do estabelecimento de valores referenciais para a atividade da ALA-D, considerado o indicador mais sensivel dos efeitos subclinicos da exposição não ocupacional ao chumbo em populações de áreas urbanas (Ingvar et al.,1997), principalmente para o controle de despejos e resíduos industriais, determinação do chumbo em alimentos, em mananciais de água e no solo, realização de avaliação epidemiológica e outras medidas de saúde pública, considerou-se relativamente pequeno o número de estudos sobre o tema, quando comparado à freqüência de trabalhos a respeito dos efeitos do metal em trabalhadores expostos.

Talvez esse fato possa ser explicado pelas dificuldades encontradas na realização de pesquisas com amostras populacionais como a necessidade de estratificação da amostragem, heterogeneidade das condições de vida das populações urbanas, utilização de critérios rigorosos de exclusão, obtenção de sujeitos considerados "saudáveis", critérios bioéticos para a colheita de amostras biológicas, disponibilidade de voluntários, entre outras variáveis.

A adoção de valores de referência de normalidade e IBMP para agentes químicos pela legislação brasileira com base em dados internacionais (Cordeiro \& Lima-Filho, 1995), também dificulta o estabelecimento de parâmetros adequados à realidade brasileira necessários à monitorização dos habitantes de áreas urbanas sujeitas a poluentes ambientais e resíduos industriais.

Conforme sugeriram Araujo et al. (1999), há necessidade de revisão da legislação brasileira que regulamenta a Patologia Clínica Ocupacional e das normas do Programa de Controle Médico de Saúde Ocupacional do Ministério do Trabalho. Isto implica na implementação de medidas para avaliação de trabalhadores que utilizam metais pesados, entre eles o chumbo, mas também na padronização do uso de bioindicadores para a monitorização das populações expostas excessivamente a riscos químicos, físicos, biológicos e psicossociais. 
MEZZAROBA, L.; TURINI, C. A.; PAOLIELO, M. M. B.; REZENDE, M. I.; MATSUO, T. Delta-aminolevulinic dehydratse acid activity in the urban population of the municipality of Londrina, Paraná. Semina: Ci. Biol. Saúde, Londrina, v. 20/21, n. 2, p. 73-79, jun. 1999/2000.

ABSTRACT: The contamination of soil, water, air and food by heavy metals, due to industrial processes, has affected not only workers but also the population in general. The determination of the activity of the $\delta$ aminolevulinic dehydratase acid (ALA-D) is the most sensitive biological indicator of the subcritic effect to lead. The purpose of this study was to assess the activity of ALA-D in a sample of an urban population in the Municipality of Londrina, Paraná. The population under study comprehended 87 subjects (52 women and 35 men), selected from na original sample of 120 volunteers and according to criteria of exclusion previously determined. The criteria excluded those individuals who are occupationally exposed to lead, with altered clinical and laboratory examinatios, obese, with hypertension, diabetes and smoking habits, and who lived close to areas where the metal is used. The reference values obtained for ALA-D activity were 21,33-59,90 U/L, with no significant differences between the values for female and male populations. These results were similar to those reported in other studies, which also used populational samples and the same analytical methodology. The standardization for the use of bio-indicators for monitoring populations exposed to environmental pollutants is one of the most useful measures for the early diagnosis and prevention of intoxication by lead in urban areas.

KEY WORDS: $\delta$-aminolevulinic dehydratase acid; environmental monitoring; lead; toxicology

\section{REFERÊNCIAS BIBLIOGRÁFICAS}

APOSTOLI, P.; MINOIA, C. Significato e utilitá dei valori di referimento in medicina del lavoro. In: IL VALORI di referimento e i valori limite nella prevenzione ambientale e occupazionale. [S.I.s.n.], 1995, p.117-130.

ARAUJO, U.C.; PIVETTA, F.R.; MOREIRA, J.C. Avaliação da exposição ocupacional ao chumbo: proposta de uma estratégia de monitoramento para prevenção dos efeitos clínicos e subclínicos. Cadernos de Saúde Pública, Rio de Janeiro, v.15, n.1, p.123-131, jan./mar. 1999.

BERLIN, A ; SCHALLER, K.H. European standardized method for the determination of $d$-aminolevulinic acid dehidratase activity in blood. Zeitschrift fuer Klinische Chemie und Klinische Biochemie, Berlin, v.12, p.389-390, 1974

BORTOLI, A. et al. Relationships between blood lead concentration and aminolevulinic acid dehydratase in alcholics and workers industrially exposed to lead. Archives of Environmental Health, v.41, n.4, p. 251-260, July/Aug., 1986.

CENTERS FOR DISEASE CONTROL. Preventing lead poisoning in young children. Atlanta: U.S. Department of Health and Human Services, 1991

CORDEIRO, R.; LIMA-FILHO, E.C. A inadequação dos valores dos limites de tolerância biológica para a prevenção da intoxicação profissional pelo chumbo no Brasil. Cadernos de Saúde Pública, Rio de Janeiro, v.11, n.2, p.177-186, abr.jun., 1995.

COSTA, C.A; TRIVELATO, G.C.; PINTO, A.M.P. et al. Correlation between plasma 5 -aminolevulinic acid concentrations and indicators of oxidative stress in lead-exposed workers. Clinical Chemistry, v.43, n.7, p.1196-1202, 1997.

DELLA ROSA H.V.; SIQUEIRA, M.E.P.B.; COLACIOPPO, S. Monitorização ambiental e biológica. In: OGA, S. Fundamentos de Toxicologia. São Paulo: Manole, 1996. p.135-151.

ELLENHORN, M.J. Medical Toxicology: diagnosis and treatment of human poisoning. 2. ed. Baltimore: Williams \& Wilkins, 1997.

FERNICOLA, N.G.G.; AZEVEDO, F.A. Niveis de chumbo e atividade da desidratase do ácido d-aminolevulínico ( $d$ ALAD) no sangue da população da Grande São Paulo, Brasil. Revista de Saúde Pública. São Paulo, v.15, p.272282, 1981

GARCIA FERNÁNDEZ, J.C. et al. Valores de referencia de aminolevulírico dehidratasa y correlación com la plombemia. Acta Bioquimica Clínica Latinoamericana. Buenos Aires, v.23, n.2, p.217-222, 1989.

IENGAR,V.; WOLTTIEZ, J. Trace elements in human clinical specimens:evaluation of literature data to identify reference values. Clinical Chemistry. v.34, p.474-481, 1988.

INGVAR, B.A. et al. Lead Binding to [delta]-Aminolevulinic Acid Dehydratase (ALAD) in Human Erythocytes. Pharmacology \& Toxicology, v.81, n.4, p.153-158, 1997.

LARINI, L. Toxicologia. 2.ed. São Paulo: Manole, 1993.

MINOIA, C. et al. Trace element reference values in tissues from habitants of European Comunity: a study of 46 elements in urine, blood and serum of italian subjects. Science Total Environmental, v.95, p.89-105, 1990.

PAOLIELO. M.M.B.et al. Valores de referência para plumbemia em população urbana. Revista de Saúde Pública. Rio de Janeiro, v.31, n.2, p.144-148, 1997

SAKAI,T.; MORITA,Y. d-aminolevulinic acid in plasma or whole blood as a sensitive indicator of lead effects, and its relation to the other heme-related parameters. Occupational and Environmental Health. v.68, n.2, p.126-132, 1996.

SALGADO, P.E.T. Biomonitorização: indicadores e limites biológicos de exposição às substâncias quimicas. São Paulo: UNESP, 1994

Toxicologia dos metais. In: OGA,S. Fundamentos de Toxicologia. São Paulo: Manole, 1996, p.153-172.

Valores de referência para metais. Revista Brasileira de Toxicologia, v.3, n.35, 1990.

SALGADO, P.E.T.; FONSECA, L.M.; LEPERA, S. Interferência do chumbo na atividade enzimática da anidrase carbônica. Revista de Ciências Farmacêuticas, São Paulo, n.7, p.6770, 1985. 
SIQUEIRA, M.E.P.B.; PESSOA, P.P.M. Reference values for daminolevulinic acid dehydratase in blood in a population of south Minas Gerais. Brazil. Revista Brasileira de Toxicologia. São Paulo, v.12, n.2, dez.,1999. suplemento.

SOUSA. M.G.F; PORTO A.P.M.;AZEVÊDO, E.S. Atividade da desidratase do ácido d-aminolevulínico (ALA-D) em uma populacão De Salvador. Bahia. Revista Brasileira de Saude Ocupacional. São Paulo. v.15, n.58, abr./maio, 1987.
WANANUKUL, $W$. et al. Lead exposure and accumulation in healthy thais: assessed by lead levels, EDTA mobilization and heme synthesis-related parameters. Journal of the Medical Association of Thailand. Bangkok, v.81,n.2, Feb. 1998.

WIDER, E.A. et al. Modificaciones de los niveles de acido delta aminolevulico dehidrasa en pacientes alcoholicos cronicos. Medicina, Buenos Aires, v.57. n.3, p.261-390. 1997. 